Revista Perspectivas Online: Biológicas \& Saúde Junho de 2017, Vol.7, no 24, p. 47-56

ISSN: 2236-8868 (Online)

DOI: $10.25242 / 886872420171165$

\title{
EFEITOS DE UM PROTOCOLO DE TREINAMENTO MENTAL ASSOCIADO À ESTIMULAÇÃO CEREBRAL PROPORCIONANDO PONTÊNCIA DOS MEMBROS INFERIORES DE ATLETAS DE VOLEIBOL
}

\author{
Luiza Pinheiro Branco ${ }^{1}$, Celby Rodrigues Vieira dos Santos ${ }^{2}$, Rodrigo Rios de Souza ${ }^{2}$ \& Mauricio \\ Rocha Calomeni ${ }^{2 *}$
}

\begin{abstract}
RESUMO
BRANCO, L.P.; SANTOS, C.R.V.; SOUZA, R.R. \& CALOMENI, M.R. Efeitos de um protocolo de treinamento mental associado à estimulação cerebral proporcionando pontência dos membros inferiores de atletas de voleibol. Perspectivas Online: Biológicas e Saúde, v.7, n.24, p. 47-56, 2017.

Introdução: No voleibol a força é uma capacidade física vital. Objetivo: Verificar se um protocolo de treinamento mental associado à estimulação cerebral melhora a potência de membros inferiores. Metodologia: 12 atletas de voleibol mulheres, 13 a 17 anos, formaram a amostra. Foi utilizado a plataforma de saltos verticais para a avaliação da potência dos membros inferiores. Os dados eletrofisiológicos coletados através de um dispositivo eletrônico chamado Procomp. O EMG foi captado por um eletrodo adesivo posicionado no ponto meso-femural direito, e, o EEG por um eletrodo monopolar posicionado no ponto $\mathrm{Cz}$, com referência nos pontos auriculares A1 e A2. A

eletrônico computadorizado capaz de produzir estímulos nas frequências de 8,12 e $15 \mathrm{~Hz}$. Por fim foi utilizado um protocolo de treinamento mental. Resultados: Não houve aumento na potência dos membros inferiores das atletas, entretanto, registrou-se aumento na média RMS do sinal do EMG no grupo experimental em relação ao controle durante $\mathrm{o}$ protocolo de treinamento mental que induzia as avaliadas a simular mentalmente situações reais do jogo, e a sugere-se que a estimulação cerebral tornou a atividade cerebral das mais homogênea, facilitando o envio de sinais do sistema nervoso central aos músculos.
\end{abstract} estimulação cerebral se deu por um aparelho

Palavras-chave: Ondas cerebrais; Estimulação Fótica, Estimulação Auditiva. 


\begin{abstract}
Introduction: in the force is a physical vital capacity. Objective: to verify if a mental training Protocol associated with brain stimulation improves the power of lower limbs. Methodology: 12 Volleyball women athletes, 13 to 17 years, formed the sample. Used vertical jumping platform for assessing the power of the lower limbs. The electrophysiological data collected through an electronic device called Procomp. The EMG was picked up by an adhesive electrode positioned in the meso-point right femoral, and the EEG by an electrode monopolar positioned at point $\mathrm{Cz}$, with reference in-ear points $\mathrm{A} 1$ and $\mathrm{A} 2$.

electronic device able to produce stimuli in the frequencies of 8,12 and $15 \mathrm{~Hz}$. Finally it was used a protocol of mental training. Results: there was no increase in power of lower limbs of athletes, however, recorded an increase in average RMS of EMG signal in the experimental group compared to the control during the mental training protocol that he the evaluated to simulate real situations of the game mentally, and suggests that brain stimulation made the brain activity of the most homogeneous, facilitating the sending of signals from the central nervous system to the muscles.
\end{abstract} Brain stimulation was given by a computerized

Keywords: Brain Waves, Photic Stimulation, Acoustic Stimulation.

\footnotetext{
${ }^{1}$ Acadêmica de Educação Física - ISECENSA - - Rua Salvador Correa, 139, Centro, Campos dos Goytacazes, RJ, CEP: 28035-310, Brasil;

${ }^{2}$ Institutos Superiores de Ensino do CENSA - ISECENSA - Laboratório de Desenvolvimento Humano e Performance Motora - LADHPEM - Rua Salvador Correa, 139, Centro, Campos dos Goytacazes, RJ, CEP: 28035-310, Brasil.

(*) e-mail: mauriciocalomeni@gmail.com

Data de chegada: 19/04/2017 Aceito para publicação: 14/06/2017
} 


\section{INTRODUÇÃO}

O treinamento mental é uma área muito estudada, pois vários estudos mostraram o quão efetivo podem ser sobre os aspectos cognitivos relacionados à performance. Nesse sentido, o treinamento mental veio com o propósito inovador de permitir a melhoria do treino físico como menos lesões e fadigas. A questão de pensar em um ato motor já é capaz de melhorar a transmissão sináptica, tornando assim um movimento mais eficiente e correto. Tal assertiva é respaldada por Rodrigues et al (2003, apud CALOMENI et al 2008) postula que a simulação mental de um determinado movimento emprega os mesmos mecanismos neurais utilizados na sua execução, evidência disso é, que quando uma pessoa pensa sobre um ato ou movimento de alguma parte do corpo, ocorre um aumento nos registros eletromiográficos na musculatura da área específica (CASTRO e SANTOS, 2007). Além disso, Franco (2000 apud ELOI, 2011) afirma que um movimento imaginado e exercitado mentalmente produz micro contrações e consequentemente uma melhora da coordenação muscular.

O voleibol é um desporto acíclico (OLIVEIRA, 1997 apud MANJI, 2013), possuindo grandes alternâncias de movimentos em sua execução. Assim sendo, aplicar treinamentos de força e potência é buscar a melhorar ações de saltos e mudanças de direções durante as partidas (RIZOLA NETO, 2004). Todas as valências físicas são essenciais para o voleibol, porém nesta pesquisa será enfatizada a potência muscular, aprimorando essa valência terá a oportunidade de elevar o nível das atletas de voleibol nas cortadas, bloqueios e saques.

A maximização da potência muscular de um atleta é fundamental para o aprimoramento do desempenho em diversas modalidades esportivas (CRONIN \& SLEIVERT, 2005 apud LAMAS et al, 2008). Para os atletas de voleibol a força é uma das principais capacidades físicas que resulta no desempenho para os gestos utilizados na modalidade.

Dessa forma, o objetivo do estudo é verificar se um protocolo de treinamento mental associado à estimulação cerebral pode melhorar a potência de membros inferiores em atletas de voleibol. Para tanto tentará responder a seguinte questão problema: Quais efeitos um protocolo de treinamento mental associado à estimulação cerebral pode proporcionar na potência de membros inferiores de atletas de voleibol?

\section{METODOLOGIA}

O projeto foi aprovado pelo Comitê de Ética em Pesquisa com Seres Humanos do ISECENSA com parecer $\mathrm{n}^{\mathrm{o}} 1.418 .428$.

A amostra foi composta por 12 indivíduos do gênero feminino, atletas de voleibol amador com idade entre 13 e 17 anos, divididos em grupo Controle (GC), n=4, e grupo Experimental (GE), n=8.

Como critério de inclusão tinham que ter pelo menos 1 ano de prática no voleibol, não possuir qualquer tipo de deficiência auditiva e estar frequentando aos treinamentos regularmente. Foram excluídas atletas que não concordarem com a participação no estudo, que tenham histórico recente de convulsões não diagnosticada.

Os dados eletrofisiológicos foram coletados utilizando um dispositivo eletrônico chamado Procomp que possui aterramento próprio o que elimina a possibilidade de interferências elétricas no sinal e aumenta a confiabilidade dos dados. O sinal eletromiográfico (EMG) foi captado por um eletrodo adesivo de superfície posicionado no ponto meso-femural da coxa direita das avaliadas, de configuração bipolar, com $20 \mathrm{~mm}$ entre as duas superfícies de detecção e um elétrodo-terra. O sinal foi convertido automaticamente em Root Mean Square (RMS), sendo coletadas 32 amostras por segundo em uma amplitude de 0 a 400 milivolts (mV). Já

Persp. online: biol. \& saúde, Campos dos Goytacazes, 24 (7), 47-56, 2017

seer.perspectivasonline.com.br 
para o sinal eletroencefalográfico (EEG) foram coletadas 256 amostras por segundo por meio de um eletrodo monopolar posicionado no ponto $\mathrm{Cz}$, com referência nos pontos auriculares $\mathrm{A} 1 \mathrm{e} \mathrm{A} 2$, como preconizado no sistema internacional 10-20.

Além desta, a potência de membros inferiores (MI) foi determinada através da plataforma de saltos verticais optical (Cefise ${ }^{\circledR}$, São Paulo) para a avaliação da potência dos membros inferiores através do salto vertical. Esse instrumento é composto por um laptop com o software "Jump System".

A estimulação cerebral via estimulação fótica e auditiva foi feita por meio de um aparelho eletrônico computadorizado capaz de produzir estímulos numa frequência predeterminada. Para esse estudo as frequências estimuladas foram 8, 12 e $15 \mathrm{Hertz}(\mathrm{Hz})$ distribuídas em intervalos pré-determinados.

O último instrumento foi um protocolo de treinamento mental que teve como objetivo induzir estados mentais propícios ao incremento na potência de membros inferiores. Nesse protocolo os primeiros 5 min foram destinados ao aumento da concentração e internalização dos pensamentos. Em seguida, nos próximos $5 \mathrm{~min}$, foi solicitado que os indivíduos se sentissem como se estivessem realizando saltos com potência cada vez maiores. Por fim, nos últimos $5 \mathrm{~min}$, os indivíduos foram induzidos a se imaginar realizando saltos em diferentes situações específicas do voleibol. Os protocolos de treinamento mental e estimulação cerebral foram aplicados simultaneamente.

Para avaliação da potência de MI foi solicitado que a avaliada simulasse um salto dado durante o movimento do bloqueio. Para isso ela foi posicionada fora da área de leitura da plataforma de saltos, sendo solicitado que a mesma desse um passo lateral para dentro dessa área e efetuasse o salto objetivando alcançar maior altura possível. Foi permitido que a avaliada executasse algumas tentativas como treinamento e aquecimento, para logo em seguida executar 3 três movimentos consecutivos, com intervalo de $10 \mathrm{~s}$ entre eles, onde foi registrado o salto com maior altura e potência.

Em seguida, para manter o caráter aleatório, foram formados através de sorteio simples os grupos Controle (GC) e Experimental (GE). Optou-se por uma divisão não homogênea dos grupos, pois, caso se confirmasse a possibilidade teórica do estudo, era do interesse do treinador da equipe que a maioria das atletas passasse pelos procedimentos experimentais.

Definidos os grupos, respeitou-se um washout de 24 hs antes do início das outras avaliações pós intervenção para se evitar possíveis efeitos agudos do procedimento de avaliação dos MI na atividade eletromiográfica das avaliadas. Ao chegar ao laboratório, cada atleta foi colocada sentada o mais confortavelmente possível para que se pudessem ser posicionados os eletrodos por onde seriam obtidos os sinais de EEG e EMG.

Os locais de fixação dos eletrodos foram devidamente higienizados com um algodão embebecido com álcool 70\%. Para fixação dos eletrodos do EEG foi utilizada uma pasta condutiva aplicada diretamente na parte de metal que faria contato com a pele, sendo fixado o eletrodo principal no ponto $\mathrm{Cz}$ por uma faixa de tecido elástico não condutivo e nos pontos auriculares A1 e A2 por fixadores de pressão. Para o sinal eletromiográfico o ponto mesofemural da perna direita foi determinado como sendo o ponto médio entre a prega inguinal e a borda superior da patela.

A coleta de base do EEG durou 1 minuto, onde foi solicitado à avaliada que permanecesse com os olhos fechados e com o mínimo de movimento possível, objetivando traçar uma linha de base da atividade cerebral. Ao término deste primeiro minuto, foi colocado na avaliada os óculos e o fone de ouvido do equipamento de estimulação fótica e auditiva, e, assim que iniciado o protocolo de treinamento mental e estimulação cerebral, também se deu início ao monitoramento da atividade eletromiográfica permitindo assim se observar seu comportamento durante as diferentes situações de treinamento mental. 
Findado os 15 minutos de estimulação cerebral associada ao treinamento mental e monitoramento da atividade eletromiográfica, foram retirados os óculos e o fone de ouvido para mais um minuto de monitoramento, desta vez da atividade eletroencefalográfica (pós-intervenção), da mesma forma que foi feito na coleta de base (EEG). Além disso, também foram repetidos os mesmos procedimentos adotados para avaliação da potência de MI.

Importante ressaltar que as atletas sorteadas para integrar o GC passaram exatamente pelos mesmos procedimentos já descritos, com a única diferença estando no momento da estimulação e treinamento mental onde o equipamento permanecia desligado e não se solicitava nenhum tipo de introspecção ou mentalização durante os 15 minutos do monitoramento através do EMG.

Como tratamento preliminar foi feita uma análise descritiva dos dados através das medidas de tendência central, e dispersão de cada variável observada nos grupos da pesquisa. Complementando a análise estatística foram aplicadas analises de variância (ANOVA) para se determinar se as possíveis diferenças encontradas apresentam significância estatística, e, caso existam tais diferenças será utilizado um teste complementar Post Hoc para se determinar onde elas vão estar. Em todas as comparações foi adotado o valor de $\mathrm{p}<0,05$ para se determinar se as diferenças são estatisticamente significantes.

\section{RESULTADOS}

A apresentação dos resultados se deu em todas as variáveis primeiramente através do resultado do teste de normalidade, em seguida pela análise descritiva e por fim pela análise inferencial intra e depois inter grupos. Dessa forma, os primeiros resultados apresentados foram sobre a variável atividade cerebral, mais especificamente sobre os registros do sinal eletroencefalográfico das ondas cerebrais Alfa e SMR coletados nos momentos pré e pós intervenção nos grupos da pesquisa.

Sobre esses dados o teste de normalidade apontou que tinham características não paramétricas, indicando que o melhor escore para representar a tendência central do grupo é a mediana. A tabela 1 traz descritivas dessa variável. 
Tabela 1. Atividade das ondas cerebrais Alfa e SMR nos momentos pré e pós intervenção nos GC e GE.

\begin{tabular}{|c|c|c|c|c|c|c|c|c|}
\hline \multicolumn{9}{|c|}{ Grupo Controle } \\
\hline & \multicolumn{4}{|c|}{ Pré-intervenção } & \multicolumn{4}{|c|}{ Pós-Intervenção } \\
\hline & \multicolumn{2}{|c|}{$\begin{array}{c}\text { Onda Cerebral } \\
\text { Alfa } \\
\end{array}$} & \multicolumn{2}{|c|}{$\begin{array}{c}\text { Onda Cerebral } \\
\text { SMR }\end{array}$} & \multicolumn{2}{|c|}{$\begin{array}{c}\text { Onda Cerebral } \\
\text { Alfa }\end{array}$} & \multicolumn{2}{|c|}{$\begin{array}{c}\text { Onda Cerebral } \\
\text { SMR }\end{array}$} \\
\hline & $\begin{array}{c}\text { Média } \\
(\mathrm{mV})\end{array}$ & $\mathrm{CV}$ & $\begin{array}{l}\text { Média } \\
(\mathrm{mV})\end{array}$ & $\mathrm{CV}$ & $\begin{array}{l}\text { Média } \\
(\mathrm{mV})\end{array}$ & $\mathrm{CV}$ & $\begin{array}{l}\text { Média } \\
(\mathrm{mV})\end{array}$ & $\mathrm{CV}$ \\
\hline Mínimo & 2,3 & 0,35 & 2,0 & 0,40 & 2,1 & 0,26 & 1,8 & 0,28 \\
\hline Mediana & 3,4 & 0,47 & 2,2 & 0,42 & 4,3 & 0,52 & 3,0 & 0,45 \\
\hline Máximo & 7,3 & 0,85 & 5,9 & 0,78 & 5,6 & 0,57 & 4,1 & 0,53 \\
\hline Desv. Pad. & 2,2 & 0,22 & 1,9 & 0,19 & 1,5 & 0,15 & 0,95 & 0,12 \\
\hline \multicolumn{9}{|c|}{ Grupo Experimental } \\
\hline & \multicolumn{4}{|c|}{ Pré-intervenção } & \multicolumn{4}{|c|}{ Pós-Intervenção } \\
\hline & \multicolumn{2}{|c|}{ Onda Cerebral Alfa } & \multicolumn{2}{|c|}{$\begin{array}{c}\text { Onda Cerebral } \\
\text { SMR }\end{array}$} & \multicolumn{2}{|c|}{$\begin{array}{c}\text { Onda Cerebral } \\
\text { Alfa }\end{array}$} & \multicolumn{2}{|c|}{$\begin{array}{c}\text { Onda Cerebral } \\
\text { SMR }\end{array}$} \\
\hline & $\begin{array}{c}\text { Média } \\
(\mathrm{mV})\end{array}$ & $\mathrm{CV}$ & $\begin{array}{c}\text { Média } \\
(\mathrm{mV})\end{array}$ & $\mathrm{CV}$ & $\begin{array}{c}\text { Média } \\
(\mathrm{mV})\end{array}$ & $\mathrm{CV}$ & $\begin{array}{c}\text { Média } \\
(\mathrm{mV})\end{array}$ & $\mathrm{CV}$ \\
\hline Mínimo & 1,2 & 0,20 & 1,6 & 0,16 & 0,86 & 0,11 & 1,2 & 0,08 \\
\hline Mediana & 3,3 & 0,50 & 3,1 & 0,39 & 2,9 & 0,35 & 3,1 & 0,24 \\
\hline Máximo & 26 & 0,90 & 17 & 0,68 & 17 & 1,4 & 16 & 1,1 \\
\hline Desv. Pad. & 8,2 & 0,28 & 5,3 & 0,18 & 5,2 & 0,41 & 4,8 & 0,34 \\
\hline
\end{tabular}

Com relação ao GC a tabela 1 evidencia que houve um aumento da atividade das ondas cerebrais Alfa e SMR do momento pré para o pós intervenção. Esse mesmo padrão se repetiu nos dados do coeficiente de variação indicando que, como era de se esperar, não houve modulação da atividade cerebral por meio do treinamento mental e estimulação fótica e auditiva. Todavia isso não ocorreu nos escores dessa variável do GE, onde se pode observar uma redução da atividade média e coeficiente de variação das ondas cerebrais Alfa de respectivamente $12 \%$ e $30 \%$, e, no coeficiente da variação das ondas SMR de $38 \%$.

Esse feito de modulação da atividade cerebral, principalmente na variabilidade da ativação dos neurônios expresso pelo coeficiente da variação, já foi registrado por Calomeni et al (2013) e relacionado a melhorias funcionais motoras em pacientes com sequelas de AVC, sendo denominado como um incremento qualitativo na atividade cerebral induzido pela estimulação cerebral. Ao que parece, quando os neurônios corticais passam a disparar de forma sincrônica permite uma maior qualidade no processamento, não necessitando assim da ativação de um número grande de neurônios para atender a demanda da tarefa. O que poderia ser considerado um incremento quantitativo na atividade cerebral. A figura 1 ilustra as observações a respeito da atividade cerebral. 

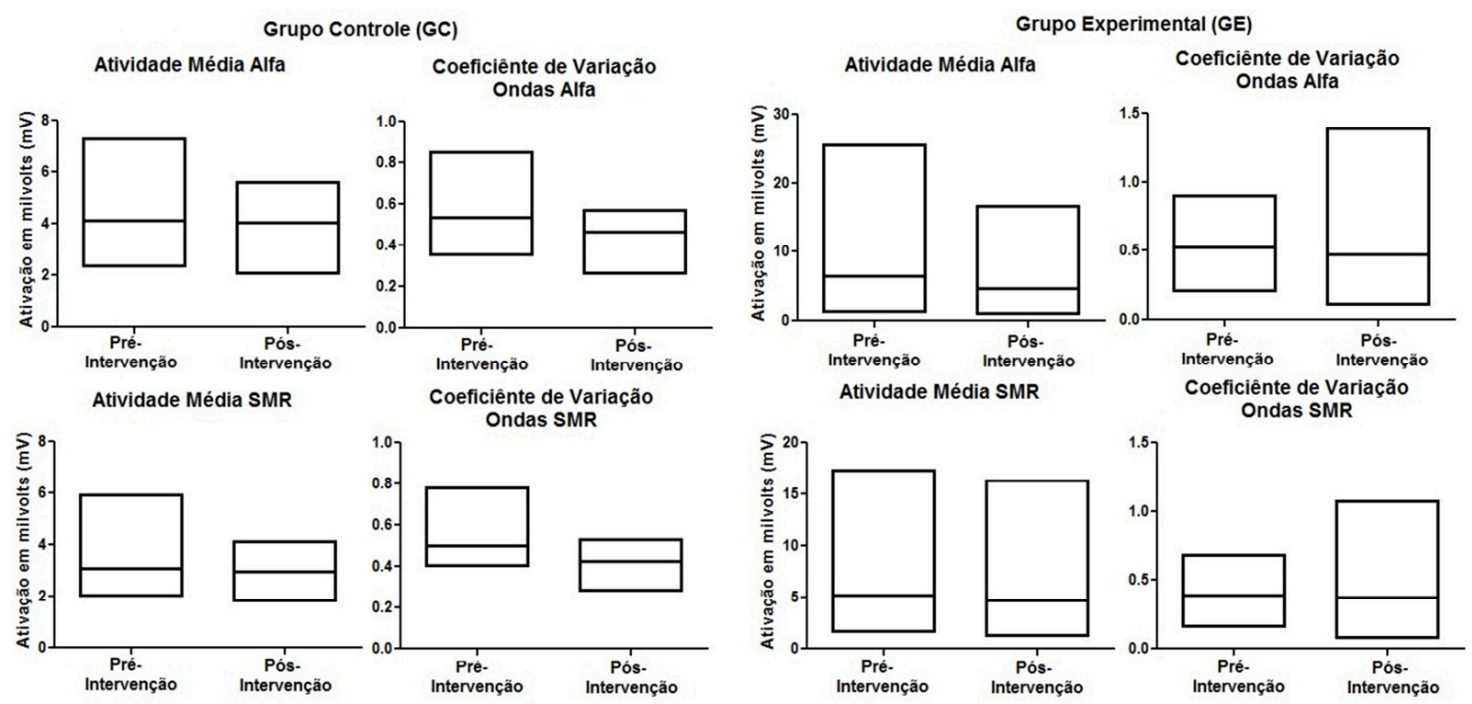

Figura 1. Plotagem dos dados do monitoramento da atividade cerebral no GC e GE nos momentos pré e pós intervenção.

Outra variável observada no estudo foi o comportamento do sinal eletromiográfico durante o período de estimulação cerebral e treinamento mental. Os dados referentes a essa observação estão apresentados na tabela 2. Nessa tabela é possível perceber como foi a média da atividade registrada no EMG durante cada fase do treinamento mental, ou seja, durante o período inicial de concentração, depois durante o período de treinamento mental através da imagética cinestésica ou sensorial, e, por fim durante o treinamento através da imagética motora.

Tabela 2. Dados do monitoramento do sinal eletromiográfico durante o período de treinamento mental e estimulação cerebral nos GC e GE.

\begin{tabular}{c|c|c|c|c|c|c}
\hline & \multicolumn{3}{|c|}{ Grupo Controle } & \multicolumn{3}{c}{ Grupo Experimental } \\
\hline & Concentração & $\begin{array}{c}\text { Imagética } \\
\text { Cinestésica }\end{array}$ & $\begin{array}{c}\text { Imagética } \\
\text { Motora }\end{array}$ & Concentração & $\begin{array}{c}\text { Imagética } \\
\text { Cinestésica }\end{array}$ & $\begin{array}{c}\text { Imagética } \\
\text { Motora }\end{array}$ \\
\hline $\begin{array}{c}\text { Intervalos de } \\
\text { Tempo }\end{array}$ & $\begin{array}{c}0-5 \\
\text { min }\end{array}$ & $\begin{array}{c}5-10 \\
\text { Min }\end{array}$ & $10-15 \mathrm{~min}$ & $\begin{array}{c}0-5 \\
\text { min }\end{array}$ & $\begin{array}{c}5-10 \\
\text { min }\end{array}$ & $\begin{array}{c}10-15 \\
\text { min }\end{array}$ \\
\hline Mínimo & $-0,14$ & $-0,14$ & $-0,20$ & 0,11 & $-0,08$ & $-0,23$ \\
\hline Mediana & $0,06^{*}$ & $0,03^{*}$ & $0,04^{*}$ & $2,3^{*}$ & $3,5^{*}$ & $4,5^{*}$ \\
\hline Máximo & 2,0 & 1,0 & 0,95 & 18 & 95 & 95 \\
\hline $\begin{array}{c}\text { Desvio } \\
\text { Padrão }\end{array}$ & 0,99 & 0,53 & 0,51 & 6,1 & 32 & 32 \\
\hline
\end{tabular}

* média RMS da atividade em $\mathrm{mV}$ durante o intervalo de tempo.

De acordo com a normalidade esses dados também apresentaram características não paramétricas, e assim como os dados do EEG será adotado a mediana como escore de tendência central para as devidas comparações. No GC percebe-se claramente que houve um padrão relativamente constante na atividade eletromiográfica durante todo o tempo de observação, o que é condizente com o fato de que essas atletas usaram o equipamento de estimulação cerebral desligado, e, também, não receberam nenhuma instrução para treinamento mental durante o tempo de coleta de dados.

Porém, quando se observa o GE nota-se claramente o efeito da estimulação associada ao treinamento mental na musculatura treinada uma vez que a média RMS do sinal do EMG do primeiro para o segundo intervalo de tempo observado aumentou $52 \%$, e, do segundo para o terceiro intervalo de tempo observado 
aumentou 29\%. Parece evidente, com base nesses números e na análise da figura 3, que a medida que o protocolo de treinamento mental induzia as avaliadas a simular mentalmente situações reais do jogo, e a estimulação cerebral tornava a atividade cerebral das mesmas mais homogênea, ou seja, mais sinais do sistema nervoso central eram enviados aos músculos e registrados pela EMG.
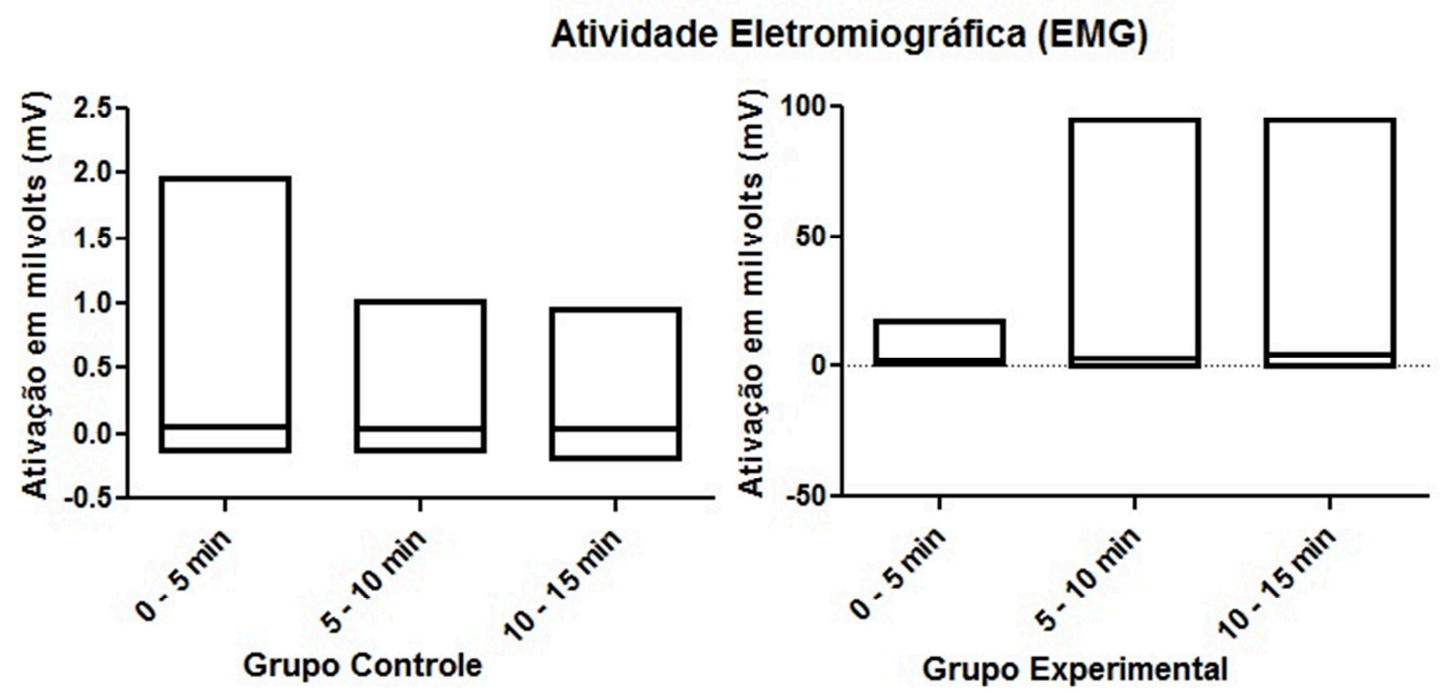

Figura 2. Dados da atividade eletromiográfica no ponto meso femural durante a aplicação do treinamento mental e estimulação cerebral no GE e intervenção placebo no GC.

A última variável do estudo que falta ser apresentada descritivamente é a potência dos membros inferiores obtidas por meio da análise da potência do salto executado no movimento de bloqueio. Os resultados dessa análise podem ser encontrados na tabela 3 , e estão ilustrados na figura 2.

Tabela 3. Apresentação descritiva dos dados relativos à avaliação da potência de MI nos GC e GE nos momentos pré e pós intervenção.

\begin{tabular}{c|c|c|c|c|c|c|c|c}
\hline & \multicolumn{4}{|c|}{ Grupo Controle } & \multicolumn{4}{c}{ Grupo Experimental } \\
\hline & \multicolumn{2}{|c|}{ Pré-intervenção } & \multicolumn{2}{c|}{ Pós-Intervenção } & \multicolumn{2}{c}{ Pré-intervenção } & \multicolumn{2}{c}{ Pós-Intervenção } \\
\hline & $\begin{array}{c}\text { Altura } \\
(\mathrm{cm})\end{array}$ & $\begin{array}{c}\text { Potência } \\
\text { /Relativa } \\
(\mathrm{W} / \mathrm{Kg})\end{array}$ & Altura $(\mathrm{cm})$ & $\begin{array}{c}\text { Potência } \\
\text { Relativa } \\
(\mathrm{W} / \mathrm{Kg})\end{array}$ & $\begin{array}{c}\text { Altura } \\
(\mathrm{cm})\end{array}$ & $\begin{array}{c}\text { Potência } \\
/ \text { Relativa } \\
(\mathrm{W} / \mathrm{Kg})\end{array}$ & $\begin{array}{c}\text { Altura } \\
(\mathrm{cm})\end{array}$ & $\begin{array}{c}\text { Potência } \\
/ \text { Relativa } \\
(\mathrm{W} / \mathrm{Kg})\end{array}$ \\
\hline Mínimo & 27 & 38 & 27 & 37 & 12 & 30 & 13 & 31 \\
\hline Máximo & 33 & 44 & 33 & 44 & 41 & 54 & 37 & 48 \\
\hline Média & 29 & 40 & 30 & 41 & 27 & 40 & 26 & 38 \\
\hline Desv. Pad. & 2,8 & 3,0 & 2,8 & 3,2 & 8,9 & 7,9 & 7,1 & 5,4 \\
\hline
\end{tabular}

A normalidade dos dados indicou que essa variável tem característica paramétrica, e dessa forma foi adotada a média aritmética como indicativo da tendência central dos grupos. Assim, se pode notar que da avaliação pré para pós intervenção, tanto no $\mathrm{GC}$ quanto no $\mathrm{GE}$, a variação média na altura do salto e na potência relativa foi muito pequena, podendo até ser considerada fruto de alguma interveniência como o empenho pessoal no teste. Pode se especular que durante o teste pré-intervenção, feito $24 \mathrm{~h}$ antes da avaliação pós-intervenção, houve um maior empenho uma vez que as mesmas apenas realizaram o salto. Todavia, no 
dia seguinte, antes da realização do salto, as atletas passaram sentadas, com movimentos reduzidos, em média por 20 minutos para coleta dos dados eletroencefalográficos e eletromiográficos, o que pode ter produzido certa desmotivação na realização do salto. As informações da tabela 3 estão ilustradas na figura 3 .

Altura do Salto

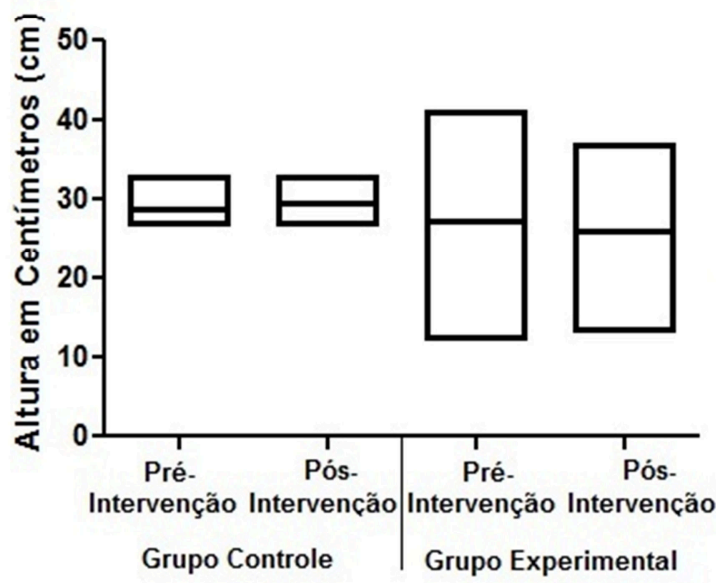

Potência Relativa

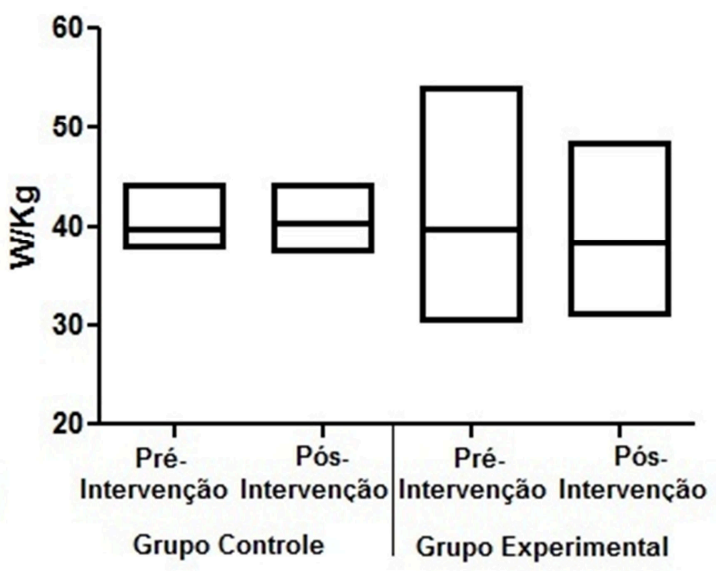

Figura 3. Dados da altura do salto e potência relativa pré e pós intervenção nos grupos Controle e Experimental.

Com relação às análises inferenciais previstas intra e inter grupos, em nenhuma delas encontrou-se valores de $\mathrm{p}<0,05$, ou seja, as diferenças apontadas na análise descritiva dos registros do EEG e EMG não se mostraram estatisticamente significantes. Porém, não se pode negar que tais diferenças existiram, e que provavelmente se deram por efeito da intervenção feita no estudo. Além disto, estudos como o de Calomeni et al (2013) verificaram que com relação à atividade cerebral, mesmo alterações estatisticamente insignificantes são suficientes para produzir ganhos funcionais significativos

Houve também em um estudo de Martins (2015) em que os resultados do EEG e do EMG não foram significativos, podendo ser pelo fato do numero de sujeitos da pesquisa ser pequeno e sendo assim reduzindo as variáveis aleatórias, além de executar os testes com apenas uma ação motora.

Também, a relação entre atividade eletromiográfica e geração de força já foi observada nos trabalhos de Silva et al (2015), os resultados obtidos na pesquisa após o treinamento relatou o aumento do desempenho cortical em um determinado grupo quando foram comparados com os grupos menos estimulados, o aumento na atividade cortical em determinadas regiões do cérebro foram relatados pelos resultados do EEG.

No estudo de Daronch (2012) Também foi capaz de perceber as diferenças existentes no EEG encontrados pelas atividades cognitivas produzidas pelos participantes do estudo.

Então, baseado nestas informações, pode se especular com certa segurança que provavelmente não se observou ganhos na altura do salto e potência relativa das atletas devido ao caráter agudo proposto nesse estudo, e, que provavelmente, a aplicação de um número maior de sessões de treinamento mental e estimulação cerebral possam produzir efeitos que alcancem diferenças estatisticamente significantes. 


\section{CONSIDERAÇÕES FINAIS}

Com base no pesquisa, os resultados demonstraram que não houve aumento na potência dos membros inferiores das atletas, confirmando assim o $\mathrm{H} 0 ; \mathrm{O}$ treinamento mental associado à estimulação cerebral não proporciona melhoria na potência de membros inferiores de atletas de voleibol.

Entretanto, de acordo com a pesquisa realizada, o efeito da estimulação associada ao treinamento mental na musculatura treinada em relação ao EMG aumentou $52 \%$ e posteriormente $29 \%$, evidenciando que o protocolo de treinamento mental induzia as avaliadas a simular mentalmente situações reais do jogo, e a estimulação cerebral tornava a atividade cerebral das mesmas mais homogêneas, mais sinais do sistema nervoso central eram enviados aos músculos e registrados pela EMG.

Sendo assim sugere-se que outros estudos sejam feitos e se aprofundem no tema proposto, para verificar os resultados aqui encontrados, em relação à melhoria da potência muscular através do treinamento mental e estimulação cerebral.

\section{REFERÊNCIAS}

CALOMENI, M. R. Estimulação audiovisual: interveniência em variáveis fisiológicas relacionadas com estresse pré-competitivo e performance competitiva. Rio de janeiro. Pg 1-114. Outubro. 2008.

CALOMENI, M.R. et al. A estimulação cerebral em pacientes com acidente vascular cerebral está associada a melhoria no desempenho motor e a incremento de eficácia na função neuronal. Arq Neuropsiquiatria. 71(3). Pg. 159-164. 2013.

CASTRO, G. G.; SANTOS, F. C. P. Treinamento mental na aprendizagem do elemento reversão simples por crianças iniciantes na ginástica artística de solo. Movimentum -Revista Digital de Educação Física Ipatinga, v.2, n.2, 11 p. ago/dez. 2007.

DARONCH, C. Medidas de EEG evocadas por tarefas de simulação de ação relativas aos treinamentos de indivíduos com habilidades percepto- motoras distintas. Porto alegre. Setembro. 2012.

MANJI, M. A. P. A influência do treinamento de força e potência na performance de jogadores de voleibol. São Paulo. Pg 1-60. 2013.

ELOI, T.S.S. Características e estruturação do treinamento mental. Artigo de revisão. Revista digital. Buenos Aires. No 161 . Outubro. 2011.

LAMAS, L. et al. Efeito de dois métodos de treinamento no desenvolvimento da força máxima e da potência muscular de membros inferiores. Revista Brasileira de Educação Física e Esporte, v. 22, n. 3, p. 235-245, 2008.

MARTINS, J. Estudo do Controle Motor Via Sincronias entre Sinais de EEG e de EMG. Outubro pg 1-77. 2015.

RIZOLA NETO, A. Uma proposta para preparação de equipes jovens de voleibol feminino. Dissertação de Mestrado. Campinas. Faculdade de Educação Física e Esporte, Universidade Estadual de Campinas, 2004.

SILVA, V.F. et al. Stimulation by Light and Sound: Therapeutics Effects in Humans. Clinical Practice \& Epidemiology in Mental Health. Volume 11. 2015.

Persp. online: biol. \& saúde, Campos dos Goytacazes, 24 (7), 47-56, 2017

seer.perspectivasonline.com.br 